\title{
Concordance Between ER, PR, HER2 neu Receptors Before and After Neoadjuvant Chemotherapy in Locally Advanced Breast Cancer
}

Research Article

Heba F. Taha, ${ }^{1 *}$ Ola M. Elfarargy, ${ }^{1}$ Reham A. Salem, ${ }^{2}$ Doaa Mandour, ${ }^{2}$

Amira A. Salem, ${ }^{3}$ Mohamed Riad ${ }^{4}$

${ }^{1}$ Medical Oncology Department, Faculty of Medicine, Zagazig University,

Zagazig, Egypt

${ }^{2}$ Clinical Oncology and Nuclear Medicine Department, Faculty of

Medicine, Zagazig University, Zagazig, Egypt

${ }^{3}$ Pathology Department, Faculty of Medicine, Zagazig University,

Zagazig, Egypt

${ }^{4}$ General Surgery Department, Faculty of Medicine, Zagazig University,

Zagazig, Egypt

Received 14 June 2019; Accepted 11 December 2019

\begin{abstract}
Background: Introducing neoadjuvant chemotherapy (NCT) in a breast cancer patient may be associated with changes in estrogen receptor (ER), progesterone receptor (PR) and human epidermal growth hormone receptor 2 (HER2) status. Patients and methods: In our prospective cohort study, we evaluated the impact of change in estrogen receptor (ER), progesterone receptor (PR) and human epidermal growth hormone receptor 2 (HER2) on the prognosis of breast cancer patients treated with neoadjuvant chemotherapy (NCT). We investigated 110 patients with locally advanced breast cancer for ER, PR and HER2 status of their lesions before and after NCT. Results: For hormone receptor status (HR) (which include ER, PR) of the residual tumor of the patients after receiving NCT, $12(10.9 \%)$ of them changed from HR (+) to HR (-) and $15(13.6 \%)$ changed from HR (-) to HR (+). For HER2 status after NCT, $8(7.3 \%)$ patients changed from HER2 $(+)$ to HER2 $(-)$ and $9(8.2 \%)$ patients changed from HER2 $(-)$ to HER2 $(+)$. Triple negative (TN) tumor phenotype changes occurred in 17 (15.5\%) patients. Patients for whom the HR status changed from positive to negative had poor prognosis for both disease-free survival (DFS) and overall survival (OS) in univariate survival analysis.

Conclusions: Changes in ER, PR, HER2 status and tumor phenotype in breast cancer patients after NCT had a negative prognostic impact and were associated with a poor prognosis.
\end{abstract}

Keywords: Breast cancer • neoadjuvant chemotherapy • concordance between ER $\bullet P R \bullet H E R 2$ neu $\bullet$ prognosis

\section{Introduction}

Breast cancer is the most frequently diagnosed life-threatening cancer in women. ${ }^{[1]}$ Neoadjuvant chemotherapy (NCT) followed by surgery is a commonly used modality for locally advanced breast cancer and is likely influenced by better outcome in these patients. ${ }^{[2]}$ Administration of NCT in breast cancer patients may be followed by changes in estrogen receptor (ER), progesterone receptor (PR) and human epidermal growth factor receptor 2 (HER2) status. Before the initiation of NCT, core-needle biopsy (CNB) is taken to confirm diagnosis and to assess the hormone receptor (HR) status (estrogen and progesterone), human epidermal growth factor receptor 2 (HER2), although this status is known to be changed after (NCT). ${ }^{[3]}$

Some studies have proved that NCT can change the status of HR and HER2, and those patients who manifested changes of HR (+) to HR (-) experienced less benefit from NCT in comparison to patients who had no changes or conversions. ${ }^{[4-7]}$ 
This study was conducted to evaluate the changes in hormone receptors, HER2, after NCT and the influence of these changes on the patient's outcome.

\section{Patients and Methods}

This study was performed at Medical Oncology Department, Clinical Oncology and Nuclear Medicine Department, Pathology Department and General Surgery Department in Zagazig University, Faculty of Medicine, Egypt, approved by the Ethical Committee in the period from December 2015 to December 2018.

We included 110 of 160 patients with locally advanced breast cancer who received NCT, 50 patients were excluded from the study as they achieved pathological complete response ( $\mathrm{pCR}$ ) and have no residual tissue for detection of change in HR and HER2 status after NCT. Patients were followed up and data were recorded in the patients' files in the shared departments; we have used the AJCC staging system for breast cancer staging, ${ }^{[9]}$ and the Nottingham (Elston-Ellis) modification of the Scarff. Bloom Richardson grading system for breast cancer (BC) grading. ${ }^{[10]}$

The primary diagnosis of invasive breast cancer of patients with locally advanced breast cancer was done by core needle biopsy (CNB) before receiving NCT to prove the diagnosis and to assess HR and HER2 status.

NCT regimens were administrated to our patients consisting of FAC (5-fluorouracil, Doxorubicin and Cyclophosphamide) every 3 weeks for 6 cycles, CEF (Cyclophosphamide, Epirubicin and 5-fluorouracil) every 3 weeks for 6 cycles, AC (Doxorubicin and Cyclophosphamide, every 3 weeks ) followed by $\mathrm{T}$ (Paclitaxel) for 12 weeks, CMF (Cyclophosphamide, Methotrexate and 5-fluorouracil) every 3 weeks for 6 cycles, AC-D (Doxorubicin, Cyclophosphamide) every 3 weeks followed by Docetaxel every 3 weeks (each for four cycles). Modified radical mastectomy within 4 weeks after NCT was done for 95 patients, while breast conserving surgery (BCS) was done for 15 patients according to the surgery team decision. Radiotherapy was applied after surgery either as a part of primary treatment in patients with BCS or as adjuvant radiotherapy.

All the patients with ER, PR positive tumors before NCT or ER, PR positive residual tumors after NCT received endocrine therapy either tamoxifen for premenopausal patients or aromatase inhibitor for postmenopausal patients for 5 years, 31 of 38 patients with HER2 (+) before NCT or HER2-positive residual tumors after NCT received trastuzumab and was completed for 1 year for all those who were indicated.
All the patients were followed up every three months, and the follow-ups ended in December 2018. The median follow-up was 27(7-36) months.

\subsection{Immunohistochemical Staining}

Immunohistochemical (IHC) analysis for ER, PR and HER2 status was performed on fresh tumor tissue that was fixed with formalin and embedded in paraffin. Tissue sections obtained for analysis were from breast tumor specimens of CNB and surgical resections before and after NCT.

ER and PR positivity were determined according to positive nuclear staining that was present at $\geq 1 \%$ of tumor cells. HER2 (+) status scoring for strong membrane staining in $\geq 10 \%$ of tumor cells were defined as $3(+)$ according to circumferential membrane staining (HercepTest; Dako Cytomation). Tumor cells with a HER2 score of $2(+)$ were further evaluated and confirmed by fluorescence in situ hybridization (FISH). [7] For IHC evaluation for ER, PR and HER2, we used: ER (M7047, clone 1D5, Dako, Produktionsvej, Glostrup, Denmark), PR (M3569, clone PgR636, Dako), HER2 (A0485, polyclonal rabbit antibody, Dako).

\subsection{Statistical Analysis}

The collected data were statistically analyzed, continuous variables were expressed as the mean \pm $\mathrm{SD}$ and median (range), and the categorical variables were expressed as a number (percentage). Continuous variables were checked for normality by using ShapiroWilk test. Mann-Whitney $U$ test was used to compare data between more than two groups of non-normally distributed variables. Disease free survival (DFS) was calculated as the time from date of surgery to relapse or the most recent follow-up in which patient was relapse free. Overall survival (OS) was calculated as the time from diagnosis to death or the most recent follow-up contact (censored). Stratification of DFS and OS was done according to different conversion groups. These time-to-event distributions were estimated using the method of Kaplan-Meier plot, and compared using twosided exact log-rank test. Cox regression analysis was performed to study the relationship between different conversion groups and both disease free survival and overall survival. A p-value $<0.05$ was considered as significant. All the statistics were performed using SPSS 20.0 for windows (SPSS Inc., Chicago, IL, USA) and MedCalc windows (MedCalc Software bvba 13, Ostend, Belgium). 


\section{Results}

\subsection{Patient Characteristics}

The detailed clinico-pathological data of our patients are fully illustrated in Table 1.

Table 1: Patients and tumor characteristics.

\begin{tabular}{|c|c|c|}
\hline \multirow[t]{2}{*}{ Characteristics } & \multicolumn{2}{|c|}{$\begin{array}{c}\text { All patients } \\
(\mathrm{N}=110)\end{array}$} \\
\hline & No. & $\%$ \\
\hline \multicolumn{3}{|l|}{ Age (years) } \\
\hline Mean $\pm S D$ & 50.84 & \pm 10.83 \\
\hline Median (Range) & 51 & $(27-73)$ \\
\hline$<35$ years & 10 & $9.1 \%$ \\
\hline$\geq 35$ years & 100 & $90.9 \%$ \\
\hline \multicolumn{3}{|l|}{ Side } \\
\hline Right breast & 47 & $42.7 \%$ \\
\hline Left breast & 63 & $75.3 \%$ \\
\hline \multicolumn{3}{|l|}{ Menopause } \\
\hline Premenopausal & 50 & $45.5 \%$ \\
\hline Postmenopausal & 60 & $54.5 \%$ \\
\hline \multicolumn{3}{|l|}{ Initial Grade } \\
\hline Grade II & 37 & $33.6 \%$ \\
\hline Grade III & 73 & $66.4 \%$ \\
\hline \multicolumn{3}{|c|}{ Pathological type } \\
\hline IDC & 93 & $84.5 \%$ \\
\hline ILC & 11 & $10 \%$ \\
\hline Other & 6 & $5.5 \%$ \\
\hline \multicolumn{3}{|l|}{ Initial T } \\
\hline $\mathrm{T} 1$ & 8 & $7.3 \%$ \\
\hline $\mathrm{T} 2$ & 32 & $29.1 \%$ \\
\hline T3 & 46 & $41.8 \%$ \\
\hline T4 & 24 & $21.8 \%$ \\
\hline \multicolumn{3}{|l|}{ Initial N } \\
\hline N1 & 30 & $27.3 \%$ \\
\hline N2 & 50 & $45.5 \%$ \\
\hline N3 & 30 & $27.3 \%$ \\
\hline \multicolumn{3}{|l|}{ Initial Stage } \\
\hline Stage IIIA & 60 & $54.5 \%$ \\
\hline Stage IIIB & 22 & $20 \%$ \\
\hline Stage IIIC & 28 & $25.5 \%$ \\
\hline \multicolumn{3}{|l|}{ Initial LVI } \\
\hline Negative & 46 & $41.8 \%$ \\
\hline Positive & 57 & $51.8 \%$ \\
\hline Unknown & 7 & $6.4 \%$ \\
\hline \multicolumn{3}{|l|}{ Initial ER status } \\
\hline Negative & 45 & $40.9 \%$ \\
\hline Positive & 65 & $59.1 \%$ \\
\hline \multicolumn{3}{|l|}{ Initial PR status } \\
\hline Negative & 52 & $47.3 \%$ \\
\hline Positive & 58 & $52.7 \%$ \\
\hline \multicolumn{3}{|c|}{ Initial HER2 status } \\
\hline+1 & 64 & $58.2 \%$ \\
\hline+2 & 23 & $20.9 \%$ \\
\hline
\end{tabular}

\subsection{Alterations in Receptor Expression}

In our study, we divided the patients according to HR and HER2 status before and after receiving NCT into groups; first group: from positive to positive, second group: from negative to negative, third group: from

\begin{tabular}{|c|c|c|}
\hline \multirow[t]{2}{*}{ Characteristics } & \multicolumn{2}{|c|}{$\begin{array}{l}\text { All patients } \\
(\mathrm{N}=110)\end{array}$} \\
\hline & No. & $\%$ \\
\hline+3 & 23 & $20.9 \%$ \\
\hline \multicolumn{3}{|l|}{ Initial HER2 FISH } \\
\hline Negative & 17 & $15.4 \%$ \\
\hline Positive & 6 & $5.5 \%$ \\
\hline \multicolumn{3}{|c|}{ Pre-NCT Tumor Phenotype } \\
\hline HER2-ve \& HR+ve & 67 & $60.9 \%$ \\
\hline $\mathrm{HER} 2+$ ve \& HR+ve & 6 & $5.5 \%$ \\
\hline HER2 + ve \& HR-ve & 13 & $11.8 \%$ \\
\hline HER2-ve \& HR-ve & 24 & $21.8 \%$ \\
\hline \multicolumn{3}{|c|}{ Neoadjuvant chemotherapy } \\
\hline CMF & 8 & $7.3 \%$ \\
\hline FAC & 44 & $40 \%$ \\
\hline FEC & 22 & $20 \%$ \\
\hline AC-T & 27 & $24.5 \%$ \\
\hline$A C-D$ & 9 & $8.2 \%$ \\
\hline \multicolumn{3}{|l|}{ Surgery } \\
\hline $\mathrm{BCS}$ & 15 & $13.6 \%$ \\
\hline MRM & 95 & $86.4 \%$ \\
\hline \multicolumn{3}{|c|}{ ER status at surgery } \\
\hline Negative & 52 & $47.3 \%$ \\
\hline Positive & 58 & $52.7 \%$ \\
\hline \multicolumn{3}{|c|}{ PR status at surgery } \\
\hline Negative & 52 & $47.3 \%$ \\
\hline Positive & 58 & $52.7 \%$ \\
\hline \multicolumn{3}{|c|}{ HER2 status at surgery } \\
\hline Negative & 8 & $7.3 \%$ \\
\hline+1 & 57 & $51.8 \%$ \\
\hline+2 & 20 & $18.2 \%$ \\
\hline+3 & 25 & $22.7 \%$ \\
\hline \multicolumn{3}{|c|}{ HER2 FISH at surgery } \\
\hline Negative & 16 & $14.6 \%$ \\
\hline Positive & 4 & $3.6 \%$ \\
\hline \multicolumn{3}{|l|}{ HR conversion } \\
\hline Negative to negative & 39 & $35.5 \%$ \\
\hline Positive to positive & 44 & $40 \%$ \\
\hline Negative to positive & 15 & $13.6 \%$ \\
\hline Positive to negative & 12 & $10.9 \%$ \\
\hline \multicolumn{3}{|l|}{ HER2 conversion } \\
\hline Negative to negative & 72 & $65.5 \%$ \\
\hline Positive to positive & 21 & $19.1 \%$ \\
\hline Positive to negative & 8 & $7.3 \%$ \\
\hline Negative to positive & 9 & $8.2 \%$ \\
\hline \multicolumn{3}{|c|}{ Tumor Phenotype Conversion } \\
\hline Concordant nTN & 53 & $48.2 \%$ \\
\hline
\end{tabular}


Table 1: Patients and tumor characteristics.

\begin{tabular}{l|cc}
\hline \multirow{2}{*}{ Characteristics } & \multicolumn{2}{|c}{$\begin{array}{c}\text { All patients } \\
\text { (N=110) }\end{array}$} \\
\cline { 2 - 3 } Concordant TN & No. & $\%$ \\
Discordant nTN & 12 & $10.9 \%$ \\
Discordant TN & 28 & $25.5 \%$ \\
Adjuvant Herceptin & 17 & $15.5 \%$ \\
No & \multicolumn{2}{|c}{} \\
Yes & 71 & $6.4 \%$ \\
Adjuvant Hormonal & & $28.1 \%$ \\
No & 39 & $35.5 \%$ \\
Yes & 71 & $64.5 \%$ \\
Radiotherapy & & \\
No & 29 & $26.4 \%$ \\
Yes & 81 & $73.6 \%$ \\
Recurrence & & \\
No recurrence & 39 & $35.5 \%$ \\
Recurrence & 71 & $64.5 \%$ \\
Mortality & & \\
Alive & 100 & $90.9 \%$ \\
Died & 10 & $9.1 \%$ \\
Follow-up duration (months) & & \\
Mean \pm SD & 24.11 & \pm 8.75 \\
Median (Range) & 27 & $(7-36)$ \\
\hline
\end{tabular}

Continuous variables were expressed as mean $\pm \mathrm{SD} \&$ median (range); categorical variables were expressed as number (percentage).

FAC (5-fluorouracil, Doxorubicin and Cyclophosphamide), CEF (Cyclophosphamide, Epirubicin and 5-fluorouracil), AC (Doxorubicin and Cyclophosphamide) followed by T (Paclitaxel; CMF (Cyclophosphamide, Methotrexate and 5-fluorouracil), AC-D (Doxorubicin, Cyclophosphamide followed by Docetaxel) HER2: human epidermal growth factor receptor 2; HR: hormone receptor; NCT: neoadjuvant chemotherapy; nTN: non-triplenegative; TN: triple-negative.

Positive to negative and fourth group :from negative to positive. The HR in 27 patients $(24.5 \%)$ was changed after NCT, 12 (10.9\%) patients with HR (+) were changed to HR (-) and 15 patients (13.6\%) showed a conversion in HR status from (-) to (+). Seventeen patients (15.5\%) showed a discordant HER2 status; 8 (7.3\%) patients were converted from HER2 $(+)$ to HER2 $(-)$, and 9 (8.2\%) were converted from HER2 (-) to HER2 (+). We classified the tumor according to their phenotypes into: HR(-)/HER2 (-), HR (+)/HER2 (-), HR (-)/HER2 $(+)$ and HR (+)/HER2 (+). Twenty-eight patients (25.5\%) developed discordance in tumor phenotypes, they were converted to non-triple negative (nTN), and 17 patients (15.5\%) were converted to TN (Table 1).

\subsection{Relation Between Changes in HR, HER2 Status and Patient Outcomes}

Survival analyses done according to Kaplan-Meier for disease-free survival (DFS) and overall survival (OS) in

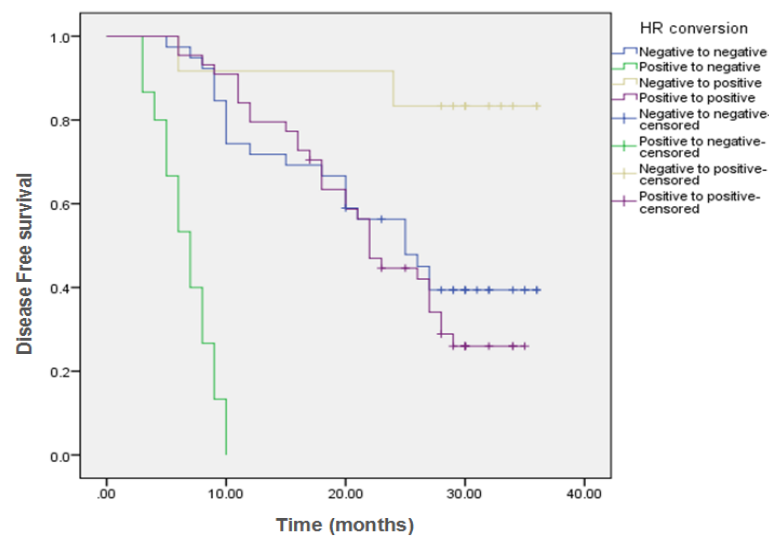

Figure (A): Kaplan Meier curve shows comparison of Disease Free Survival (DFS) in HR conversion group.

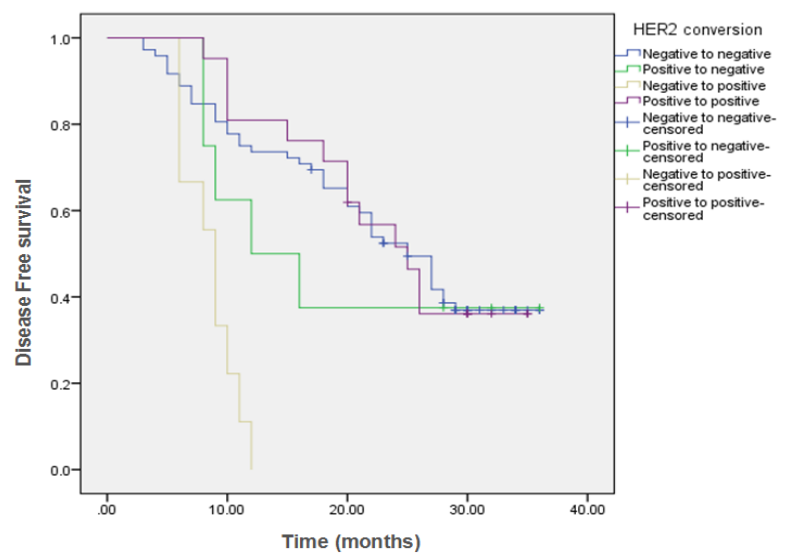

Figure (B): Kaplan Meier curve shows comparison of Disease Free Survival (DFS) in HER2 conversion group.

the four groups according to HR and HER2 status are shown in Tables 2 and 3, and Figures A and B. DFS and OS were significantly worse in patients who had conversion in the HR status from $(+)$ to $(-)$ after NCT than the other three groups of patients $(P<0.001),(P$ $<0.001$ ), respectively (Figures $A$ and $2 A$ ). Patients who had HER2 (-) before and after NCT had better DFS rather than those who were converted from HER2 $(-)$ to HER 2(+) $(P<0.001)$ (Figure B), but no significant difference was observed in OS $(P=0.365)$ (Figure $2 \mathrm{~B})$.

We evaluated the difference in patients' survival according to the changes in HR and HER2 status after NCT by univariate Cox regression analyses for DFS and OS

(Table 4), HR and HER2 conversion were statistically significant $(P<0.001),(P<0.001)$ respectively for DFS only but not significant for OS, HR $(P=0.127)$ and HER2 ( $p=0.557$ ). The groups of patients who were converted to $\mathrm{HR}(-)$ had significantly worse survival outcomes (Hazard ratio, $\mathrm{HR}=12.606, P<0.001$ for DFS; but not for OS HR $=578737.963, P=0.924)$ in 
Table 2: Comparison of disease Free Survival (DFS) in the different conversion groups.

\begin{tabular}{|c|c|c|c|c|c|c|c|c|}
\hline \multirow{2}{*}{ Group } & \multirow{2}{*}{$\begin{array}{c}\text { Total } \\
\mathbf{N}\end{array}$} & \multirow{2}{*}{$\begin{array}{c}\mathbf{N} \text { of } \\
\text { events }\end{array}$} & \multirow{2}{*}{$\begin{array}{c}\text { Censored } \\
\text { N (\%) }\end{array}$} & \multicolumn{4}{|c|}{ Disease Free Survival (DFS) } & \multirow{2}{*}{ p-valuet } \\
\hline & & & & Median & 1-year & 2-year & 3-year & \\
\hline \multicolumn{9}{|l|}{ HR conversion } \\
\hline Negative to negative & 39 & 23 & $16(41 \%)$ & 25 months & $71.8 \%$ & $45 \%$ & $39.4 \%$ & $<0.001$ \\
\hline Positive to negative & 15 & 15 & $0(0 \%)$ & 7 months & $0 \%$ & ---- & ---- & \\
\hline Negative to positive & 12 & 2 & $10(83.3 \%)$ & NR & $91.7 \%$ & $83.3 \%$ & $83.3 \%$ & \\
\hline Positive to positive & 44 & 31 & $13(29.5 \%)$ & 22 months & $79.5 \%$ & $44.6 \%$ & $26 \%$ & \\
\hline \multicolumn{9}{|l|}{ HER2 conversion } \\
\hline Negative to negative & 72 & 44 & $28(38.9 \%)$ & 25 months & $73.6 \%$ & $69.4 \%$ & $36.9 \%$ & $<0.001$ \\
\hline Positive to negative & 8 & 5 & $3(37.5 \%)$ & 12 months & $50 \%$ & $37.5 \%$ & $37.5 \%$ & \\
\hline Negative to positive & 9 & 9 & $0(0 \%)$ & 9 months & $0 \%$ & --- & --- & \\
\hline Positive to positive & 21 & 13 & $8(38.1 \%)$ & 25 months & $81 \%$ & $51.6 \%$ & --- & \\
\hline \multicolumn{9}{|c|}{ Tumor Phenotype Conversion } \\
\hline Concordant nTN & 53 & 29 & $24(45.3 \%)$ & 27 months & $84.9 \%$ & $65.2 \%$ & ---- & $<0.001$ \\
\hline Concordant TN & 12 & 7 & $5(41.7 \%)$ & 20 months & $66.7 \%$ & $50 \%$ & $41.7 \%$ & \\
\hline Discordant nTN & 28 & 25 & $3(10.7 \%)$ & 9 months & $21.4 \%$ & $14.3 \%$ & ---- & \\
\hline Discordant TN & 17 & 10 & $7(41.2 \%)$ & 22 months & $58.8 \%$ & $47.1 \%$ & $41.2 \%$ & \\
\hline
\end{tabular}

Categorical variables were expressed as percentage; continuous variables were expressed as median; NR: not reached yet; $\uparrow$ Log rank test; $p<0.05$ is significant; HER2: human epidermal growth factor receptor 2; HR: hormone receptor.

Table 3: Comparison of Overall Survival (OS) in the different conversion groups.

\begin{tabular}{|c|c|c|c|c|c|c|c|c|}
\hline \multirow{2}{*}{ Group } & \multirow{2}{*}{$\begin{array}{c}\text { Total } \\
\mathbf{N}\end{array}$} & \multirow{2}{*}{$\begin{array}{c}\mathbf{N} \text { of } \\
\text { events }\end{array}$} & \multirow{2}{*}{$\begin{array}{c}\text { Censored } \\
\text { N (\%) }\end{array}$} & \multicolumn{4}{|c|}{ Overall Survival (OS) } & \multirow{2}{*}{ p-valuet } \\
\hline & & & & Median & 1-year & 2-year & 3-year & \\
\hline \multicolumn{9}{|l|}{ HR conversion } \\
\hline Negative to negative & 39 & 5 & $34(87.2 \%)$ & NR & $97.4 \%$ & $89.4 \%$ & $85.7 \%$ & $<0.001$ \\
\hline Positive to negative & 15 & 4 & $11(73.3 \%)$ & ---- & $69.1 \%$ & --- & --- & \\
\hline Negative to positive & 12 & 1 & $11(91.7 \%)$ & NR & $91.7 \%$ & $91.7 \%$ & $91.7 \%$ & \\
\hline Positive to positive & 44 & 0 & $44(100 \%)$ & NR & $100 \%$ & $100 \%$ & $100 \%$ & \\
\hline \multicolumn{9}{|l|}{ HER2 conversion } \\
\hline Negative to negative & 72 & 5 & $67(93.1 \%)$ & NR & $94.3 \%$ & $92.7 \%$ & $92.7 \%$ & 0.365 \\
\hline Positive to negative & 8 & 0 & $8(100 \%)$ & NR & $100 \%$ & $100 \%$ & $100 \%$ & \\
\hline Negative to positive & 9 & 1 & 8 (88.9\%) & ---- & $88.9 \%$ & $88.9 \%$ & --- & \\
\hline Positive to positive & 21 & 4 & $17(81 \%)$ & NR & $90.5 \%$ & $85.4 \%$ & $79.3 \%$ & \\
\hline \multicolumn{9}{|c|}{ Tumor Phenotype Conversion } \\
\hline Concordant nTN & 53 & 3 & $50(94.3 \%)$ & NR & $98.1 \%$ & $96.2 \%$ & $93.9 \%$ & 0.219 \\
\hline Concordant TN & 12 & 2 & $10(83.3 \%)$ & NR & $90.9 \%$ & $81.8 \%$ & $81.8 \%$ & \\
\hline Discordant nTN & 28 & 4 & $24(85.7 \%)$ & ---- & $84.8 \%$ & $84.8 \%$ & ---- & \\
\hline Discordant TN & 17 & 1 & $16(94.1 \%)$ & NR & $94.1 \%$ & $94.1 \%$ & $94.1 \%$ & \\
\hline
\end{tabular}

Categorical variables were expressed as percentage; continuous variables were expressed as median; NR: not reached yet; $\uparrow$ Log rank test; $p<0.05$ is significant; HER2: human epidermal growth factor receptor 2; HR: hormone receptor. 
Table 4: Univariate Cox regression analysis for Disease Free Survival and Overall Survival.

\begin{tabular}{|c|c|c|c|c|c|c|c|}
\hline \multirow{2}{*}{ Group } & \multirow{2}{*}{$\begin{array}{l}\text { Total } \\
\mathbf{N}(\%)\end{array}$} & \multicolumn{3}{|c|}{ DFS } & \multicolumn{3}{|c|}{ OS } \\
\hline & & $\mathrm{Haz}$ & R $\quad(95 \% \mathrm{Cl})$ & p-value & HazR & $(95 \% \mathrm{Cl})$ & p-value \\
\hline HR conversion & & & & $<0.001$ & & & 0.127 \\
\hline Negative to negative & $39(35.5 \%)$ & 0.818 & $(0.477-1.405)$ & 0.467 & 114437.447 & & 0.933 \\
\hline Positive to negative & $15(13.6 \%)$ & 12.606 & $(5.762-27.581)$ & $<0.001$ & 578737.963 & & 0.924 \\
\hline Negative to positive & $12(10.9 \%)$ & 0.159 & $(0.038-0.667)$ & 0.012 & 67638.402 & & 0.936 \\
\hline Positive to positive & $44(40 \%)$ & & 1.000 & & 1.000 & & \\
\hline HER2 conversion & & & & $<0.001$ & & & 0.557 \\
\hline Negative to negative & $72(65.5 \%)$ & 1.023 & $(0.551-1.901)$ & 0.943 & 0.381 & $(0.102-1.420)$ & 0.151 \\
\hline Positive to negative & $8(7.3 \%)$ & 1.295 & $(0.461-3.638)$ & 0.624 & 0.000 & & 0.987 \\
\hline Negative to positive & $9(8.2 \%)$ & 6.104 & $(2.461-15.136)$ & $<0.001$ & 0.650 & $(0.072-5.864)$ & 0.701 \\
\hline Positive to positive & $21(19.1 \%)$ & & 1.000 & & 1.000 & & \\
\hline Tumor Phenotype Conversion & & & & $<0.001$ & & & 0.276 \\
\hline Concordant nTN & $53(48.2 \%)$ & & 1.000 & & 1.000 & & \\
\hline Concordant TN & 12 (10.9\%) & 1.298 & $(0.568-2.966)$ & 0.536 & 3.557 & $(0.593-21.328)$ & 0.165 \\
\hline Discordant nTN & 28 (25.5\%) & 4.548 & $(2.617-7.904)$ & $<0.001$ & 3.771 & $(0.823-17.280)$ & 0.087 \\
\hline Discordant TN & 17 (15.5\%) & 1.376 & $(0.670-2.826)$ & 0.385 & 1.100 & $(0.114-10.577)$ & 0.935 \\
\hline
\end{tabular}

HazR: Hazards Ratio; 95\%Cl: 95\%Confidence Interval; DFS: disease-free survival; HER2: human epidermal growth factor receptor 2; HR: hormone receptor; nTN: non-triple-negative; OS: overall survival; TN: triple-negative

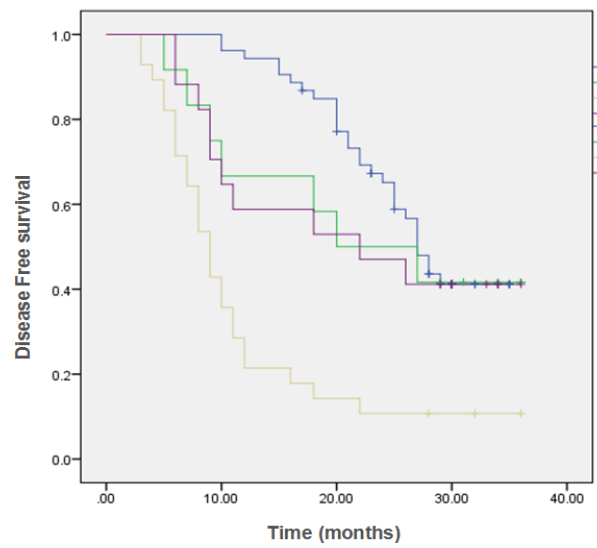

Figure (C): Kaplan Meier curve shows comparison of Disease Free Survival (DFS) in Tumor Phenotype Conversion group.

comparison to the positive patients with a concordant HR status ( $\mathrm{HR}=1$ for DFS and OS), and patients who were converted to $\mathrm{HR}(+)(\mathrm{HR}=0.159, P=0.012$ for DFS; but not significant, $P=0.936$ for OS). The change in HER2 status from positive to negative alone had no significant worse outcome for DFS or OS.

\subsection{Discordance in Tumor Phenotype and Patient Outcomes}

Kaplan-Meier analyses were done for tumor phenotype conversion (Figure C). Patients who had no changes in

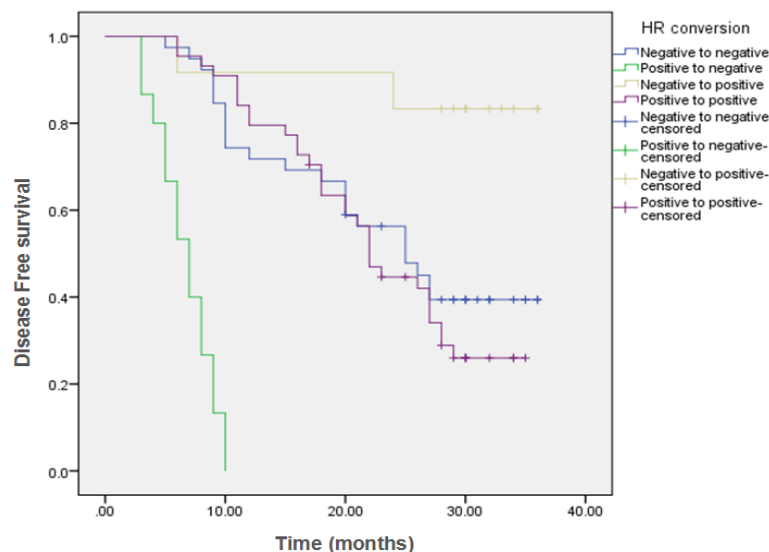

Figure (2A): Kaplan Meier curve shows comparison of Overall Survival (OS) in HR conversion group.

the tumor phenotype had better outcomes in comparison to the discordant cases $(P<0.001$ for DFS but not significant $P=0.219$ for OS) (Figures $C$ and $2 \mathrm{C}$ ).

Patients with discordant nTN tumor phenotype (the tumor phenotype was changed) had significantly worse DFS (HR $=4.548, P<0.001)$ but not significant for OS (HR $=3.771, P=0.087)$ in comparison to patients with concordant TN (the tumor phenotype was TN and unchanged) $(H R=1.298, p=0.536$ for DFS and $H R=$ 3.557, $p=0.165$ for OS) and discordant TN (the tumor phenotype was changed) $(\mathrm{HR}=1.376, P=0.385$ for DFS, $\mathrm{HR}=1.100, P=0.935$ for OS ) in univariate analyses (Table 4, Figures $C$ and $2 \mathrm{C}$ ). 


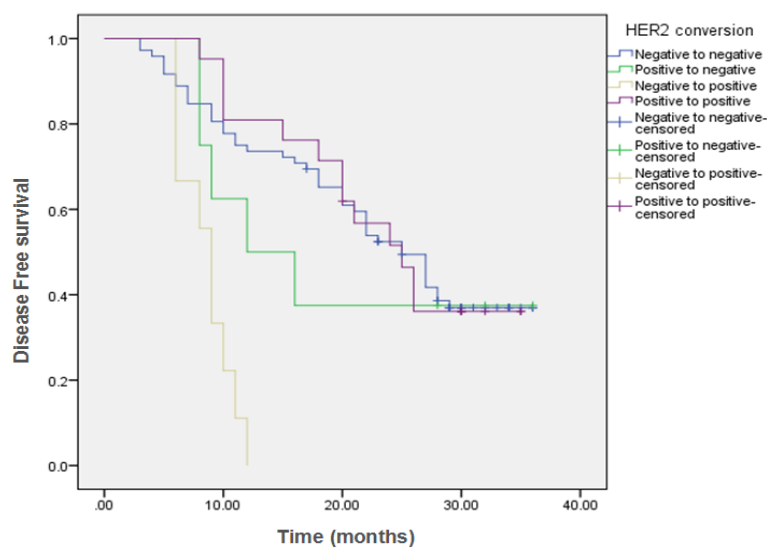

Figure (2B): Kaplan Meier curve shows comparison of Overall Survival (OS) in HER2 conversion group.

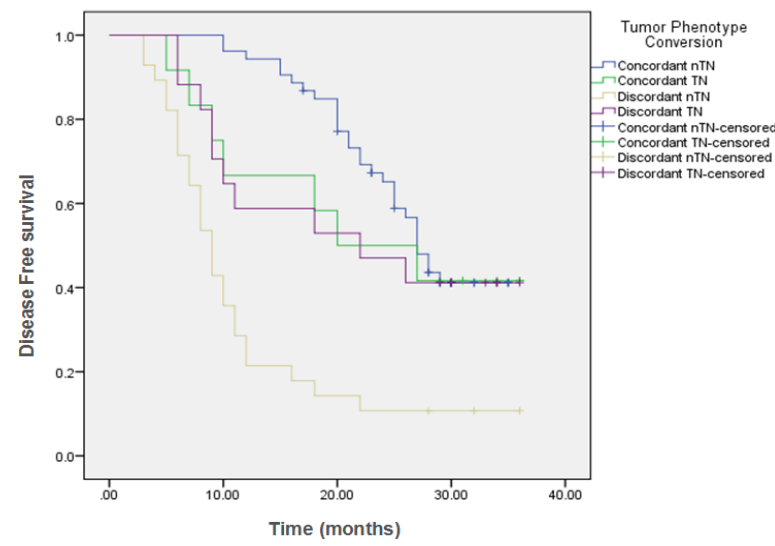

Figure (2C): Kaplan Meier curve shows comparison of Overall Survival (OS) in Tumor Phenotype Conversion group.

\section{Discussion}

The result of conversion of HR and HER2 status after NCT in breast cancer patients still have conflicting data. ${ }^{[8]}$

Locally advanced breast cancer was a problem in the past years, but with the development of neoadjuvant chemotherapy, the patients now can have a better control of the disease. ${ }^{[6-12]}$

In our prospective cohort study, we described that patients with locally advanced breast cancer whose HR were changed from HR (+) to HR (-) after NCT had a worse prognosis, while the effect of HER2 conversion alone did not have a significant effect on the prognosis.

$\mathrm{Xi}$ Jin et al. reported that HR conversion from positive to negative had worse disease-free survival (DFS) and overall survival (OS) in multivariate survival analysis. ${ }^{[16]}$ Chen et al. found that patients for whom HR changed from positive to negative after NCT had negative impact in DFS and OS. ${ }^{[7]}$
Hirata et al. (2009) did not find a significant difference in DFS and OS between the HR-positive group and HRconversion group. ${ }^{[15]}$ However, in our study, patients who had been converted from HR (+) to $(-)$ after NCT had significantly worse DFS $(P<0.001)$ and worse OS $(P$ $<0.001)$.

The mechanism of HR and HER2 status conversion after NCT is complicated. The intratumoral heterogeneity may be due to the presence of different types of clones with different phenotypes within the same individual tumors. ${ }^{[13-14]}$

Bines et al. and Rose et al. mentioned that ovarian function and adrenal glands can be suppressed by chemotherapy, ${ }^{[18-19]}$ and the change of HR status from (+) to (-) after NCT may be due to decrease in the hormones caused by this suppression. ${ }^{[11]}$ This mechanism of suppression might be the main cause for HR conversion from (+) to (-) after NCT.

Niikura et al. (2016) reported that the change in HER2 status regardless of pre-NCT HR status, had different results $(21.4 \%$ for HER2 positive-to-negative change and $3.4 \%$ for HER2 negative-to-positive change) from our study, where $8(7.3 \%)$ of the patients were changed from HER2 (+) to HER2 (-), and $9(8.2 \%)$ changed from HER2 (-) to HER2 (+). ${ }^{[20]}$ In our study, in HER2 conversion groups, patients who remained HER2 (-) after NCT had better DFS rather than those who were converted from HER2 $(-)$ to HER2 $(+)(P<0.001)$, but no significant difference was observed in OS $(P=0.365)$. In spite of the conversion of HER2 status after NCT, this conversion was not significant when compared with patients who did not receive NCT. ${ }^{[12]}$

Our study focused on the discordance of tumor phenotype and HR status before and after NCT and the effect on patient's prognosis.

We have several limitations in our study. First, the patients didn't receive the same regimens of NCT, so we can't decide if receptor conversion is due to special or multiple factors. Some studies mentioned that patients with high level of $\mathrm{Ki}-67$ expression that had reduction after NCT had better prognosis. ${ }^{[21-22]}$ In our study, we did not mention the effect of reduction of Ki-67 expression on patients' prognosis. Future studies will be required to identify the prognostic value of receptor conversion.

\section{Summary and Conclusion}

Our prospective cohort study indicated the presence of discordance in HR, HER2 status and loss of receptor positivity after NCT had poor outcome and negative prognostic impact. These results might help for the selection of adjuvant therapy and improve patients 
outcome and survival. NCT may be the direct cause of tumor phenotype conversion with the changes in tumor receptors but the mechanism of conversion needs further studies.

\section{Recommendation}

Because of discordance between HR and HER2 expressions in primary diagnostic and residual breast tissue after NCT, it is helpful to retest the HR and HER2 status after NCT as this may have a prognostic value and may help in the selection of subgroup of patients who need further evaluation of treatment plan.

\section{References}

[1] Torre LA, Bray F, Siegel RL, Ferlay J, Lortet-Tieulent J, Jemal A. Global cancer statistics, 2012. CA Cancer J Clin. 2015 Mar. 65(2): 87-108.

[2] Mauri D, Pavlidis N, loannidis JPA. Neoadjuvant Versus Adjuvant Systemic Treatment in Breast Cancer: A Meta-Analysis. J Natl Cancer Inst. 2005; 97: 188-194.

[3] Bartlett JM1, Brookes CL, Robson T, van de Velde CJ, Billingham LJ, Campbell FM, et al.Estrogen receptor and progesterone receptor as predictive biomarkers of response to endocrine therapy: a prospectively powered pathology study in the Tamoxifen and Exemestane Adjuvant Multinational trial. J Clin Oncol. 2011; 29: 1531-1538.

[4] Zhang N, Moran MS, Huo Q, Haffty BG, Yang Q. The hormonal receptor status in breast cancer can be altered by neoadjuvant chemotherapy: a metaanalysis. Cancer Invest. 2011; 29: 594-598.

[5] Burcombe RJ, Makris A, Richman PI, Daley FM, Noble S, Pittam M, et al. Evaluation of ER, PgR, HER2 and $\mathrm{Ki}-67$ as predictors of response to neoadjuvant anthracycline chemotherapy for operable breast cancer. Br J Cancer. 2005; 92: 147-155.

[6] Shet T, Agrawal A, Chinoy R, Havaldar R, Parmar $\mathrm{V}$, Badwe R. Changes in the tumor grade and biological markers in locally advanced breast cancer after chemotherapy-implications for a pathologist. Breast J. 2007; 13: 457-464.

[7] Chen S, Chen CM, Yu KD, Zhou RJ, Shao ZM. Prognostic value of a positive-to-negative change in hormone receptor status after neoadjuvant chemotherapy in patients with hormone receptorpositive breast cancer. Ann Surg Oncol. 2012; 19: 3002-3011.

[8] HER-2/neu expression in locally advanced breast carcinomas: pre-and post-neoadjuvant chemotherapy. Quddus RM, Sung JC, Zhang C, Pasqueriello T, Eklund M, Steinhoff MM Breast Cancer. 2005; 12(4): 294-8.

[9] Giuliano AE1, Connolly JL2, Edge SB3, Mittendorf EA4, Rugo HS5, Solin LJ6, et al. (2017) Breast Cancer-Major changes in the American Joint Committee on Cancer eighth edition cancer staging manual. CA Cancer J Clin 67: 290-303.

[10] Elston CW, Ellis IO (2002) Pathological prognostic factors in breast cancer I. The value of histological grade in breast cancer: experience from a large study with long-term follow-up. Histopathology 19 : 403-410.

[11] van de Ven S, Smit VT, Dekker TJ, Nortier JW, Kroep JR. Discordances in ER, PR and HER2 receptors after neoadjuvant chemotherapy in breast cancer. Cancer Treat Rev. 2011; 37: 422-430.

[12] Zhang N, Moran MS, Huo Q, Haffty BG, Yang Q. The hormonal receptor status in breast cancer can be altered by neoadjuvant chemotherapy: a metaanalysis. Cancer Invest. 2011; 29: 594-598.

[13] Bertos NR, Park M. Breast cancer - one term, many entities? J Clin Invest. 2011; 121: 3789-3796.

[14] Dent R, Trudeau M, Pritchard KI, Hanna WM, Kahn HK, Sawka CA, et al. Triple-negative breast cancer: clinical features and patterns of recurrence. Clin Cancer Res. 2007; 13: 4429-4434.

[15] Hirata T1, Shimizu C, Yonemori K, Hirakawa A, Kouno $\mathrm{T}$, Tamura $\mathrm{K}$, et al. Change in the hormone receptor status following administration of neoadjuvant chemotherapy and its impact on the long-term outcome in patients with primary breast cancer. Br J Cancer. 2009; 101: 1529-1536. doi: 10.1038/sj.bjc.6605360.

[16] Xi Jin1, Yi-Zhou Jiang1, Sheng Chen1, Ke-Da Yu1, Zhi-Ming Shao1, Gen-Hong Di. Prognostic 
value of receptor conversion after neoadjuvant chemotherapy in breast cancer patients: a prospective observational study. Oncotarget, Vol. 6, No. 11, 2015.

[17] Chen S, Chen CM, Yu KD, Zhou RJ, Shao ZM. Prognostic value of a positive-to-negative change in hormone receptor status after neoadjuvant chemotherapy in patients with hormone receptorpositive breast cancer. Ann Surg Oncol. 2012; 19: 3002-3011. doi: 10.1245/s10434-012-2318-2.

[18] Bines J, Oleske DM, Cobleigh MA. Ovarian function in premenopausal women treated with adjuvant chemotherapy for breast cancer. J Clin Oncol. 1996; 14: 1718-1729.

[19] Rose DP, Davis TE. Effects of Adjuvant Chemohormonal Therapy on the Ovarian and Adrenal Function of Breast Cancer Patients. Cancer Res. 1980; 40: 4037-4047.

[20] Niikura N, Tomotaki A, Miyata H, Iwamoto T, Kawai $\mathrm{M}$, Anan $\mathrm{K}$, et al. Changes in tumor expression of HER2 and hormone receptors status after neoadjuvant chemotherapy in 21,755 patients from the Japanese breast cancer registry. Ann Oncol. 2016; 27: 480-487. doi: 10.1093/annonc/mdv611.

[21] Matsubara N1, Mukai $H$, Masumoto $M$, Sasaki M, Naito Y, Fujii S, Wada N. Survival outcome and reduction rate of $\mathrm{Ki}-67$ between pre- and post-neoadjuvant chemotherapy in breast cancer patients with non-pCR. Breast Cancer Res Treat. 2014; 147: 95-102. doi: 10.1007/s10549014-3084-6.

[22] Matsubara N, Mukai H, Fujii S, Wada N. Different prognostic significance of $\mathrm{Ki}-67$ change between pre- and post-neoadjuvant chemotherapy in various subtypes of breast cancer. Breast Cancer Res Treat. 2013; 137: 203-212. doi: 10.1007/s10549012-2344-6. 